\title{
ARTICULAR CHANGES ASSOCIATED WITH INTERNAL DERANGEMENT OF THE KNEE
}

\author{
T. TASKER, W. WAUGH
}

\author{
From the Department of Surgery, Queen's Medical Centre, Nottingham
}

\begin{abstract}
A prospective study was made of 100 knees which had been operated on for various kinds of internal derangement. An observer, who did not know the clinical condition of the knee, recorded whether or not there were articular lesions of the femoral condyles. Lesions were found to be localised to a triangular area on the medial condyle and to a strip on the lateral condyle. Similar lesions have been described in association with flexion deformities in rheumatoid and osteoarthritic knees.

Lesions were found in $\mathbf{4 9}$ out of $\mathbf{5 0}$ knees which had been locked for more than three days. They were also found in 29 knees in which there appeared to be full extension before operation, but where there was either a torn meniscus with a history of intermittent locking or serious anterolateral instability. In these cases it was considered that the final "screw-home" mechanism of full extension had been lost. Lesions were not present in 10 knees which appeared locked, but in nine of these the interval between injury and operation was less than three days. Lesions were not found in eight knees where there was full extension and no history of locking or instability. In four knees with a plica syndrome similar lesions were present.
\end{abstract}

An association between a particular pattern of loss of articular cartilage and the presence of a flexion deformity has been demonstrated in rheumatoid and osteoarthritic knees (Waugh, Newton and Tew 1980). Erosion, and subsequent osteophyte formation, was recognised in a triangular area on the medial femoral condyle and on a strip of the lateral femoral condyle (Fig. 1). These areas are in contact with the anterior horns of the medial and lateral menisci when the knee is fully extended (Brantigan and Voshell 1941). The base of the triangular area on the medial femoral condyle represents

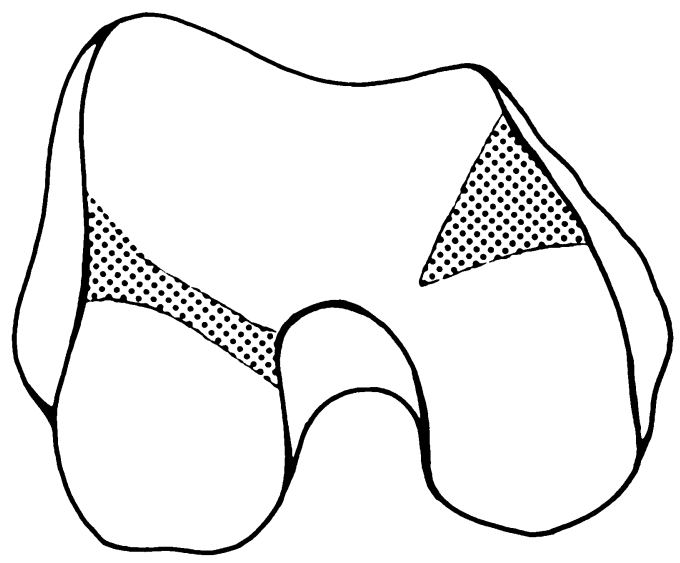

Fig. 1

Diagram showing the site of the medial triangular lesion and the lateral strip lesion. the extent of rotation which occurs as the knee locks in full extension - the "screw-home" mechanism. We propose that an internal derangement, such as a torn meniscus, which results in the loss of full extension will also lead to similar lesions in specific areas of the femoral condyles. Random observations by one of us (WW) suggested that there was often an infiltration of vascular synovium over the triangular area of the medial condyle in a knee locked by a bucket-handle tear of the medial meniscus. It therefore seemed reasonable to make a systematic study to determine whether or not this association was significant.

\section{MATERIAL AND METHOD}

One of the authors (TT) observed and recorded the findings in 100 unselected arthrotomies carried out for mechanical disorders at Harlow Wood Orthopaedic Hospital (65 operations), Leicester Royal Infirmary (12 operations) and Leicester General Hospital (23 operations); TT had no knowledge of the diagnosis and physical findings at the time of the operation. Details of the patients including the history and clinical examination before operation and the operative findings-particularly whether a lesion of the femoral condyle on the side of the arthrotomy was found-were recorded on a pro forma; special attention was paid to the surgeon's assessment of whether or not there was a block to full extension.

Sixty-five of the patients were men ( 74 knees) and 23 were women (26 knees). The age range was from 17 to 61 years with an average of 27.9 years. There were 64 right knees and 36 left knees. The diagnoses and the side of the arthrotomy are given in Table I. Fifty-nine knees had limited extension and were considered to be locked at the time of operation.

T. Tasker, FRCS Ed, Senior Registrar

W. Waugh, MA, MChir, FRCS, Professor of Orthopaedic and Accident Surgery

Department of Surgery, Medical School, Queen's Medical Centre, Clifton Boulevard, Nottingham NG7 2UH, England.

Requests for reprints should be sent to Professor W. Waugh.

(C) 1982 British Editorial Society of Bone and Joint Surgery 0301-620X/82/4090-0486 \$2.00 
Table I. Diagnosis of $100 \cdot$ knees and operative approach used for arthrotomy

\begin{tabular}{|c|c|c|c|}
\hline \multirow[b]{2}{*}{ Diagnosis } & \multirow{2}{*}{$\begin{array}{c}\text { Number of } \\
\text { knees }\end{array}$} & \multicolumn{2}{|c|}{ Side of arthrotomy } \\
\hline & & Medial & Lateral \\
\hline Torn meniscus & 47 & 32 & 15 \\
\hline Ligamentous injuries & 14 & 6 & 8 \\
\hline $\begin{array}{l}\text { Combined meniscal and } \\
\text { ligamentous injuries }\end{array}$ & 27 & 3 & 24 \\
\hline Loose bodies & 3 & 1 & 2 \\
\hline Chondromalacia patellae & 5 & 5 & 0 \\
\hline Plica syndrome & 4 & 4 & 0 \\
\hline Total & 100 & 51 & 49 \\
\hline
\end{tabular}

\section{RESULTS}

A lesion of the articular cartilage was recorded as being present when it was seen in either the medial triangular area or in the lateral strip, depending on which side of the knee was being explored. A vascular layer of synovium was sometimes found spreading from the edge of the condyle which could be "brushed off" the underlying articular cartilage with a swab (Fig. 2). In other cases the synovium was adherent and the cartilage showed macroscopic signs of degeneration. Although it obviously was not possible to observe any one knee at different times, it is likely that the lesion occurs progressively, and finally there would be complete loss of articular cartilage and formation of osteophytes in the areas described. The extent of the pathological condition was recorded, but we have only been concerned in this study with its presence or its absence.

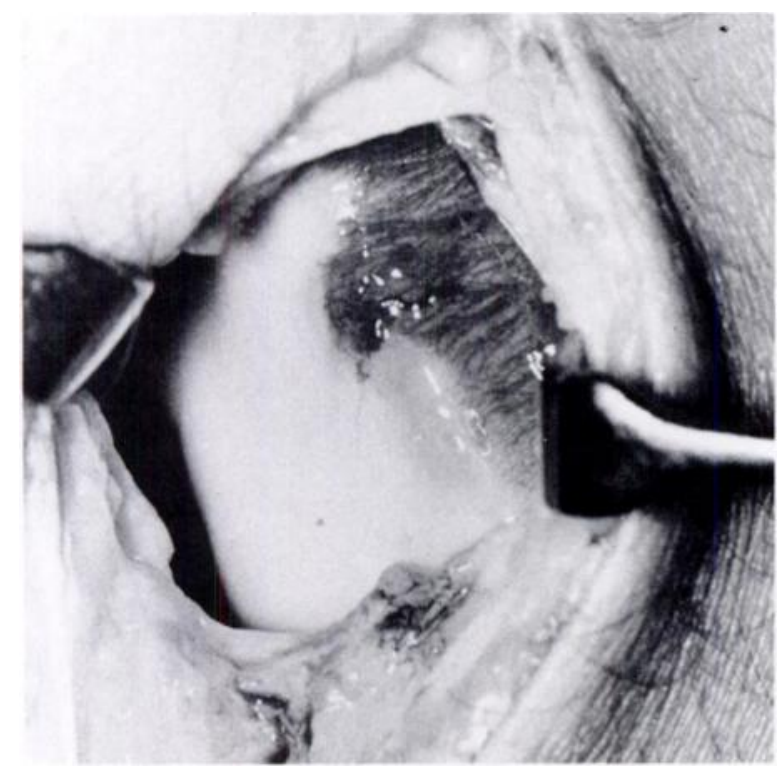

Fig. 2

Synovial vascular infiltration is seen at an early stage spreading into the triangular area on the medial femoral condyle in a locked knee.
The knees operated on were divided into four groups depending on whether or not extension was limited and whether an articular cartilage lesion was present.

Group 1. This group consisted of 49 knees, all with a block to full extension and a lesion of the articular cartilage. It was not possible to demonstrate a definite relationship between the length of time which the knee had been locked and the extent of the lesion, but in eight patients who were operated on between the third and fifth day after injury, the invading vascular synovium could be "brushed off" the underlying articular cartilage. Group 2. This group consisted of 10 knees which were locked before operation but in which the characteristic lesion was not found. However, nine of the operations were carried out within $\mathbf{4 8}$ hours of the injury.

Group 3. This group comprised the 29 knees that were not considered to be locked at the time of operation, but in which a lesion of the articular cartilage was present. All of the knees had evidence of ligamentous instability (8), or a torn meniscus (9) or both (12); some knees also had a history of intermittent locking before operation.

Group 4. There were eight knees in Group 4 which had full extension and in which no lesion was present. Three of these had meniscal tears, but no history of locking. The remaining knees were diagnosed as having chondromalacia patellae. The relative rarity of diagnosis of this condition in the series was because, more often than not, the diagnosis was made by arthroscopy and the knee was not opened.

Finally, there were four knees in the series which needed separate consideration and were not placed in one of the four groups. In these there was no block to extension and a medial plica was found. A lesion of the articular cartilage was present, but was more extensive than the triangular area of the medial condyle.

\section{DISCUSSION}

The findings of the knees in Groups 1 and 4 supported the hypothesis that a lesion of the articular cartilage is found in those knees where there is a block to full extension and not when the knee can be extended fully. These findings were in keeping with the results described in rheumatoid and osteoarthritic knees (Waugh et al. 1980). Results in Groups 2 and 3 did not appear to support the hypothesis. However, in Group 2, nine out of the ten knees with limited extension were explored within three days of the injury, and this interval may have been too short to allow the typical lesion to develop. The results in Group 3 were more interesting because the lesion was found in knees that apparently could be fully extended, but in each of these knees there was either a torn meniscus with a history of intermittent locking (nine) or there was disabling instability, which was usually anterolateral; a MacIntosh fasciodesis was carried out in 19 of the unstable knees. Loss of the normal "screwhome" mechanism occurs if there is a deficient anterior 
cruciate ligament (Ellison 1980) and this is likely to be the case whenever clinical instability is present (Jones 1980). Frankel, Burstein and Brooks (1971) demonstrated that any internal derangement was likely to limit the axial rotation associated with the "screw-home" mechanism and it therefore seems reasonable to conclude that the knees in Group 3 were likely to have lost the lateral rotation of the tibia on the femur which takes place at the end of full extension. This cannot be recognised clinically and may occur in a knee which appears to extend normally.

Thus it becomes necessary to reconsider our premise and to restate the hypothesis: the characteristic lesions of articular cartilage are likely to be associated with a block to extension of more than three days duration and in knees where there is rotatory instability or a history of locking.

There certainly is no doubt that the synovial vascular infiltration is frequently seen in knees with an internal derangement, and it seems reasonable to associate this with the loss of full extension and with the loss of the normal "screw-home" mechanism. It certainly is difficult to see how the infiltration could occur if the area was in contact with an opposing articular surface, and it is easy to understand how the converse could be true. But whatever produces the lesion, two important practical considerations arise. First, if the articular lesion is seen during arthroscopy or at arthrotomy and if a meniscal tear is not at once apparent, a very careful search must be made. The results demonstrated in this paper lend support to the empirical teaching of some experienced orthopaedic surgeons in this respect.

The second matter concerns the development of osteoarthritis in knees with meniscal tears or instability. It does seem that if a knee is locked for any length of time changes which can lead to osteoarthritis will occur on the triangular and strip areas of the femoral condyles. Experimental evidence has shown that articular cartilage needs mechanical stimulation for normal nutrition of chondrocytes (Ekholm and Norbäck 1951). Furthermore, Salter and McNeil (1965) and Salter et al. (1980) demonstrated that degenerative changes occur in the articular cartilage of a rabbit's knee with a flexion deformity. It seems reasonable to suppose that in human knees degenerative changes will occur in the triangular and strip areas which lose contact with their opposing articular surfaces as soon as the normal mechanism of full extension is lost.

Whilst there are good functional reasons for dealing urgently with a locked knee, it is also true that no knee should be left with any degree of loss of extension (or instability) since this will lead to osteoarthritis. This, of course, is not the only mechanism which can operate to produce progressive osteoarthritis in knees with meniscal injuries or ligamentous instability.

In conclusion, synovial infiltration, and subsequent articular cartilage degeneration, will occur in the triangular and strip areas on the medial and lateral femoral condyles in a knee where there is a block to full extension and when the final "screw-home" mechanism is lost.

We wish to thank the orthopaedic surgeons at Harlow Wood Orthopaedic Hospital and at the Leicester Hospitals who allowed us to include their patients and observe their operations.

\section{REFERENCES}

Brantigan OC, Voshell AF. The mechanics of the ligaments and menisci of the knee joint. J Bone Joint Surg 1941:23:44-66.

Ekholm R, Norbäck B. On the relationship between articular changes and function. Acta Orthop Scand $1951 ; 21: 81-98$.

Ellison AE. The pathogenesis and treatment of anterolateral rotatory instability. Clin Orthop 1980;147:51-5.

Frankel VH, Burstein AH, Brooks DB. Biomechanics of internal derangement of the knee: pathomechanics as determined by analysis of the instant centers of motion. J Bone Joint Surg [ Am] 1971;53-A:945-62.

Jones KG. Results of use of the central one-third of the patellar ligament to compensate for anterior cruciate ligament deficiency. Clin Orthop $1980 ; 147: 39-44$

Salter RB, McNeil R. Pathological changes in articular cartilage secondary to persistent joint deformity. J Bone Joint Surg [Br] $1965 ; 47-B: 185-6$.

Salter RB, Simmonds DF, Malcolm BW, Rumble EJ, MacMichael D, Clements ND. The biological effect of continuous passive motion on the healing of full-thickness defects in articular cartilage. J Bone Joint Surg [Am] 1980;62-A:1232-51.

Waugh W, Newton G, Tew M. Articular changes associated with a flexion deformity in rheumatoid and osteoarthritic knees. J Bone Joint Surg $[\mathrm{Br}] 1980 ; 62-\mathrm{B}: 180-3$. 